\title{
Comparison of Stereotactic Body Radiotherapy Delivery Techniques for Early-Stage Lung Cancer Using Lung Toxicity Modeling
}

\author{
Chunhui Han, Timothy E. Schultheiss, Jeffrey Y. C. Wong \\ Department of Radiation Oncology, City of Hope National Medical Center, Duarte, CA, USA \\ Email:chan@coh.org
}

How to cite this paper: Han, C.H., Schultheiss, T.E. and Wong, J.Y.C. (2018) Comparison of Stereotactic Body Radiotherapy Delivery Techniques for Early-Stage Lung Cancer Using Lung Toxicity Modeling. International Journal of Medical Physics, Clinical Engineering and Radiation Onco$\log$, 7, 1-14.

https://doi.org/10.4236/ijmpcero.2018.71001

Received: November 2, 2017

Accepted: January 21, 2018

Published: January 24, 2018

Copyright $\odot 2018$ by authors and Scientific Research Publishing Inc. This work is licensed under the Creative Commons Attribution International License (CC BY 4.0).

http://creativecommons.org/licenses/by/4.0/

\begin{abstract}
Purpose: Lung toxicity is a primary side effect in stereotactic radiotherapy (SBRT) for early-stage non-small cell lung cancer (NSCLC). We aimed to use a set of radiobiological models to evaluate and compare modern IMRT delivery techniques with three-dimensional conformal techniques for SBRT treatment of NSCLC in terms of lung toxicity, and aimed to compare the results from different radiobiologcal models. Methods: Ten early-stage NSCLC patients treated with SBRT were retrospectively selected. Five treatment plans were generated to deliver $50 \mathrm{~Gy}$ in five fractions to the planning target volume for each case: a helical tomotherapy (HT) plan, two three-dimensional cofnromal radiotherapy (3D-CRT) plans using 6-MV and 10-MV photon beams respectively, and two volumetric modulated arc therapy (VMAT) plans using one and two arc fields respectively. The lung RDV was calculated with three parallel functional sub-unit (FSU) models and two normal tissue complication probability (NTCP) models. Results: Both the HT and VMAT plans showed significantly higher contralateral mean lung dose and lower ipsilateral mean lung dose compared to the 3D-CRT plans. There was no statistically significant difference in terms of lung toxicities between the IMRT and 3D-CRT techniques using either the FSU models or the NTCP models. Based on both the FSU and the NTCP models, there was strong correlation between lung toxicity and the mean lung dose in SBRT treatment plans. Conclusions: Based on both the NTCP and parallel FSU models, both IMRT and traditional 3D-CRT delivery techniques could achieve comparable lung sparing inn SBRT treatment of early-stage lung cancer. However, the validity of the radiobiological model results should be checked by clinical data.
\end{abstract}

\section{Keywords}

Early-Stage Lung Cancer, SBRT, Stereotactic Body Radiotherapy, Lung Toxicity 


\section{Introduction}

In recent years, hypofractionated stereotactic body radiotherapy (SBRT) has been widely implemented as a definitive treatment modality for early-stage non-small cell lung cancer (NSCLC), especially for patients who are not candidates for surgery due to existing morbidities including cardiopulmonary complications [1] [2] [3]. In a systematic review of thirty-five published studies, the local control rate was all above $80 \%$ at 1 - 5 years for stage I NSCLC treated with SBRT, and a significant number of patients did not have any sign of adverse effects during the course of treatment [4]. While early-stage lung cancer patients could potentially be cured from SBRT treatments, it is imperative to minimize radiation-induced toxicities to reduce post-treatment morbidities. As multiple radiation therapy (RT) delivery techniques exist to deliver SBRT to the lung, including three-dimensional conformal radiotherapy (3D-CRT), and intensitymodulated radiotherapy (IMRT) techniques, objective and quantitative methods to evaluate treatment plan quality are needed to choose the optimal treatment plan for disease control and sparing of normal organs.

In SBRT treatment of lung cancer, normal lung toxicity is a primary concern for treatment complications [4]. In conventional lung cancer RT, commonly used dosimetric quantities include the volume of the lung receiving dose above 20 Gy (V20), and the mean lung dose (MLD) [5]. Due to the large dose per fraction in SBRT treatments, clinical experiences based on conventional RT fractionation schemes may not be applicable in SBRT treatment plan evaluation [6]. More sophisticated radiobiological models have been proposed to predict radiation toxicities to the lung. The equivalent uniform dose (EUD) model has long been used to evaluate organ toxicities. In the EUD model, a power-law formula is used to convert the dose-volume histogram (DVH) from a RT plan to a single equivalent dose parameter, which can be translated to normal tissue complication probability (NTCP) through a sigmoid function [7]. On the other hand, the DVH can be reduced to a volume parameter using mathematical formulations based on the parallel functional sub-unit (FSU) model [8] [9]. In contrast to the EUD concept, the parallel FSU model quantifies the percentage of lung volume damaged by RT treatment, which is potentially a clinically measurable quantity. Previous studies using the parallel FSU model indicate that this model could be relevant in evaluation of organ toxicities in RT treatments [10] [11].

In this study, we aimed to use the existing lung toxicity models to quantify lung toxicities for comparison of different RT delivery techniques. We also aimed to evaluate the discrepancies between existing radiobiological models in lung toxicity modeling in SBRT treatments.

\section{Materials and Methods}

We retrospectively selected ten patients with early-stage non-small cell lung cancer who previously received definitive SBRT treatment at our institution. Table 1 lists patient characteristics. Prior to treatment planning, each patient 
Table 1. Characteristics of patients in this study.

\begin{tabular}{cccc}
\hline Patient Index & Age at treatment & PTV/cc & PTV Location \\
\hline 1 & 70 & 30.2 & RUL \\
2 & 72 & 104.6 & LLL \\
3 & 81 & 40.4 & LLL \\
4 & 70 & 206.5 & RUL \\
5 & 72 & 93.3 & RML \\
6 & 92 & 187.9 & LUL \\
7 & 62 & 30.3 & RLL \\
8 & 54 & 38.6 & LLL \\
9 & 78 & 143.0 & RLL \\
10 & 64 & 36.4 & RUL \\
\hline
\end{tabular}

received computed tomography (CT) scans in the thoracic region with $3 \mathrm{~mm}$ slice thickness in the helical mode. Three CT scans were performed for each patient, while the patient was in shallow free breathing and at the end of the inspiration and expiration phases, respectively. In a treatment planning system (Eclipse Version 11, Varian Medical Systems, Inc., Palo Alto, California), the three CT image sets were registered rigidly, and contours of critical organs including the lungs, heart, spinal cord, esophagus were drawn on the free-breathing CT images. The gross target volume (GTV) was drawn on each of the CT image sets, and then combined to form the internal target volume (ITV). The planning target volume (PTV) was created by adding $5-\mathrm{mm}$ lateral margin and $10-\mathrm{mm}$ superior-inferior margin to the ITV. The average PTV volume was $91.1 \pm 67.7 \mathrm{~cm}^{3}$ (range: $30.2-206.5 \mathrm{~cm}^{3}$ ).

In this study, we generated the following five treatment plans for each patient:

1) A $3 D-C R T$ treatment plan using $6 M V$ photon beams (3D-6MV plan). Each plan used 12 non-coplanar fields, with the orientation and relative weighting of each field manually chosen for each individual plan to minimize critical organ dose. In the Eclipse treatment planning system, the analytical anisotropic algorithm (AAA) was used for dosimetric calculation with heterogeneity correction applied.

2) A $3 \mathrm{D}-\mathrm{CRT}$ plan using $10 \mathrm{MV}$ photon beams (3D-10MV plan). For each case, the $3 \mathrm{D}-10 \mathrm{MV}$ plan used the same field geometry as the $3 \mathrm{D}-6 \mathrm{MV}$ plan. The same dosimetric calculation algorithm was used.

3) A helical tomotherapy plan (HT plan). The jaw size of either $2.5 \mathrm{~cm}$ or 1.0 $\mathrm{cm}$ was used, depending on PTV dimension. A pitch of 0.15 was used in all the HT plans. A superposition-convolution algorithm with heterogeneity correction was used for dose calculation.

4) A single-arc RapidArc VMAT plan (VMAT-1 plan). In this plan, a 6-MV single arc field rotates around the patient for a complete gantry rotation. Both the dose rate and the gantry speed were allowed to modulate during the arc rotation. The Progressive Resolution Optimization (PRO) algorithm in the Eclipse 
treatment planning system was used for optimization, and the AAA algorithm was used for final dose calculation with heterogeneity correction.

5) A two-arc RapidArc VMAT plan (VMAT-2 plan). In this plan, two 6-MV arc fields, with identical complete gantry rotation range but opposing rotational directions, were used. The same optimization and dose calculation algorithms were used as in the VMAT-1 plans.

The RTOG Protocol 0623 was followed as the guideline for dose prescription and normal organ dose constraints. The PTV receives 50 Gy in 5 uniform fractions in each treatment plan. All the treatment plans were normalized so that 95\% of the PTV received at least the prescription dose. In IMRT treatment plan optimization, higher priority was on minimizing normal lung dose, rather than on dose homogeneity of the PTV.

Lung toxicity was evaluated using the parallel FSU model in the following steps. First, the dose-volume histogram (DVH) of the normal lung volume in the differential form was converted to the normalized biologic equivalent (NBE) DVH using the linear-quadratic model with the $\alpha / \beta$ ratio of $3 \mathrm{~Gy}$ for the normal lung [6] [12] [13]. Specifically, the dose in the $i$-th bin of the lung DVH is normalized using the following equation:

$$
D_{i, \text { normalized }}=D_{i} \cdot \frac{1+\left(D_{i} / n\right) / 3 G y}{1+2 G y / 3 G y} \text {, where } n \text { is the number of treatment fractions. }
$$

Second, the normalized DVH was reduced to a single lung toxicity parameter that represents the relative damaged volume (RDV) for the lung. To calculate the RDV for each treatment plan, a local effective function, $E(D)$, was calculated as a function of the local lung dose. The RDV is then given by [6] [11]:

$$
\mathrm{RDV}=\sum_{i} E\left(D_{i, \text { normalized }}\right) \cdot V_{i}
$$

where $D_{i \text { normalized }}$ and $V_{i}$ are the normalized dose and percentage volume in the $i$-th bin of the differential DVH, respectively. This definition is based on the assumption that the lung is composed of parallel functional sub-units with identical radiation response characteristics.

Multiple mathematical formulations exist in the literature for calculating the effective dose function $E(D)$. When $E(D)$ is a linear function of the local dose $\mathrm{D}$, $\mathrm{RDV}$ is mathematically equivalent to using the MLD in plan evaluation. Other authors used sigmoid forms for the calculation of $E(D)$. To evaluate the robustness and consistency of the models, we applied the following three mathematical formulations in this study.

1) The logistic model formulation [11]. With this model, $E(D)$ is given by a logistic function: $E(D)=\frac{1}{1+\left(\frac{D_{\mathrm{L} 50}}{D}\right)^{k}} \cdot D_{\mathrm{L} 50}$ is the dose level at which the local dose effect is $50 \%\left(E\left(D_{\mathrm{L} 50}\right)=50 \%\right)$, and $k$ represents the steepness of the logistic function curve. In this study, $k$ is taken as 2 .

2) The S-shape model formulation [6]. With this model, $E(D)$ is given by: 


$$
E(D)= \begin{cases}\frac{\frac{D}{D_{\mathrm{L} 50}}-1}{1+\left(\frac{D}{D_{\mathrm{L} 50}}-1\right)^{2}}+1 / 2, & \text { when } D \leq 2 D_{\mathrm{L} 50} \\ 1, & \text { when } D>2 D_{\mathrm{L} 50}\end{cases}
$$

3) The modified linear model formulation. $E(D)$ is given by:

$$
E(D)= \begin{cases}\frac{D}{2 D_{\mathrm{L} 50}}, & \text { when } D \leq 2 D_{\mathrm{L} 50} \\ 1, & \text { when } D>2 D_{\mathrm{L} 50}\end{cases}
$$

Figure 1 plots the three local effective dose functions for comparison. While $E(D)$ approaches 1 asymptotically with increasing dose in the logistic model, it reaches 1 when $D=2 D_{\mathrm{L} 50}$ in both the S-shape model and the modified linear model.

In published literature, the value of $D_{\mathrm{L} 50}$ had significant uncertainty due to heterogeneity in datasets used and toxicity level evaluated [5]. To evaluate the robustness of the parallel FSU model, we allowed $D_{\mathrm{L} 50}$ to vary from 20 Gy to 40 Gy in the analysis. For comparison, we also calculated normal tissue complication probability (NTCP) of the total lung for each treatment plan, based on the Lyman-Kutcher-Burman (LKB) model as well as the mean-lung-dose (MLD) model, respectively. With the LKB model, the EUD of the normal lung is given by:

$$
\mathrm{EUD}=\left(\sum_{i} D_{i}^{1 / n} \frac{V_{i}}{V_{\text {total }}}\right)^{n}
$$

and the NTCP value is obtained by:

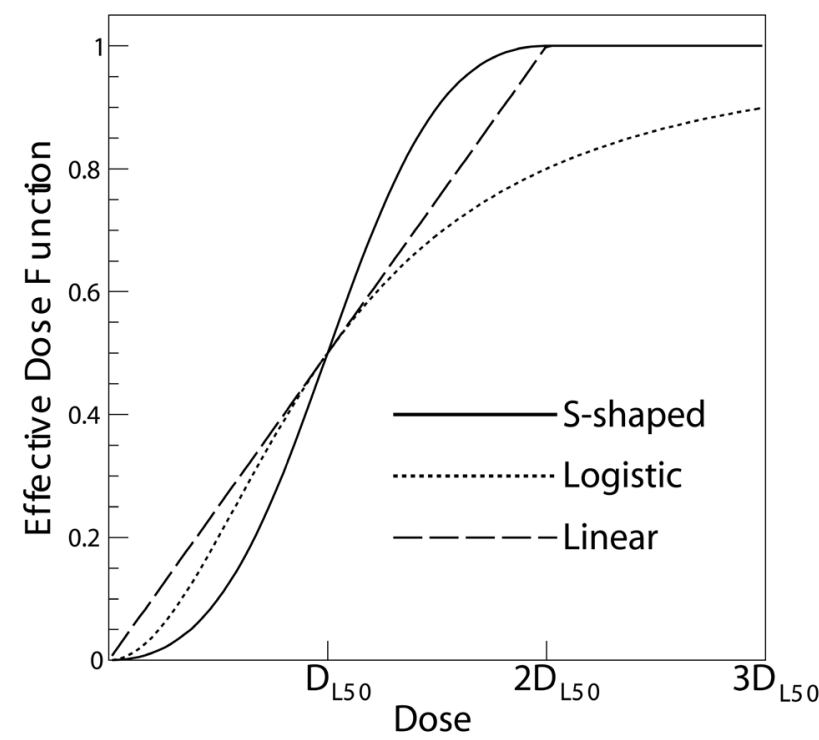

Figure 1. Three local effective dose functions used in this study: the S-shaped function (solid line), the logistic function (dotted line), and the linear function (dashed line). 


$$
\mathrm{NTCP}=\frac{1}{\sqrt{2 \pi}} \int_{-\infty}^{t} \mathrm{e}^{-\frac{x^{2}}{2}} \mathrm{~d} x \text {, where } t=\frac{\mathrm{EUD}-\mathrm{TD}_{50}}{m \cdot \mathrm{TD}_{50}} .
$$

Three parameters, $n, m$, and $\mathrm{TD}_{50}$, exist in the $\mathrm{LKB}$ model.

With the MLD formulation, the NTCP can be expressed by a logistic function:

$$
\mathrm{NTCP}=\frac{\mathrm{e}^{b_{0}+b_{1} \cdot \mathrm{MLD}}}{1+\mathrm{e}^{b_{0}+b_{1} \cdot \mathrm{MLD}}}
$$

Parameters for the LKB and MLD models were obtained from [5], based on clinical data for radiation pneumonitis.

Correlation between the mean lung dose and the modeling outcomes (RDV or NTCP values) was evaluated using Spearman's rank order correlation analysis, using the statistical computing system R [14].

\section{Results}

Table 2 lists average and standard deviation values for the maximum PTV dose and mean PTV dose for each treatment technique. On average, the maximum dose to the PTV was about $34 \%$ higher relative to the prescription dose in the $3 \mathrm{D}-6 \mathrm{MV}$ and 3D-10MV plans; it was $23 \%-24 \%$ higher in the HT, VMAT-1, and VMAT-2 plans. Paired t-tests showed that both the maximum dose and the mean dose to the PTV were significantly higher in the 3D-6MV and 3D-10MV plans compared to the IMRT plans (two tailed p-value $<0.05$ ). There was no statistically significant difference in the maximum dose or the mean dose between the 3D-6MV and 3D-10MV plans, or among the HT, VMAT-1, and VMAT-2 plans.

Table 3 lists average values of the mean dose to the ipsilateral and contralateral lungs, mean dose to the heart, and the maximum dose to the spinal cord with each treatment technique. Paired t-tests were performed to evaluate the statistical significance in difference for each dosimetric parameter between any pair of treatment techniques. There was no significant difference in the maximum dose to the spinal cord or the mean dose to the heart among all the treatment techniques. As to the contralateral lung, each of the three IMRT techniques showed significantly higher mean lung dose compared to the 3D techniques, while there was no significant difference among the three IMRT techniques. For the ipsilateral lung, each of the three IMRT techniques showed significantly

Table 2. PTV dose statistics for each treatment technique.

\begin{tabular}{ccc}
\hline Treatment Delivery Technique & Average $D_{\max } \pm$ StdDev/Gy & Average $D_{\text {mean }} \pm$ StdDev/Gy \\
\hline 3D-6MV & $67.0 \pm 3.7$ (range: $61.9-73.3$ ) & $57.3 \pm 1.6$ (range: $54.3-59.5$ ) \\
3D-10MV & $67.1 \pm 4.4$ (range: $59.7-74.3$ ) & $58.9 \pm 1.8$ (range: $54.7-61.1$ ) \\
HT & $61.3 \pm 2.2$ (range: $57.3-64.7$ ) & $58.8 \pm 2.0$ (range: $54.3-61.3$ ) \\
VMAT-1 & $61.8 \pm 2.6$ (range: $58.6-67.7$ ) & $55.2 \pm 1.5$ (range: $52.3-57.9$ ) \\
VMAT-2 & $62.0 \pm 2.3$ (range: $59.3-67.2$ ) & $55.6 \pm 1.1$ (range: $54.1-57.7$ ) \\
\hline
\end{tabular}

$D_{\text {max }}:$ maximum dose; $D_{\text {mean }}$ : mean dose; StdDev: standard deviation. 
Table 3. Dosimetric statistics for major organs.

\begin{tabular}{cccccc}
\hline Treatment Technique & HT & 3D-6MV & 3D-10MV & VMAT-1 & VMAT-2 \\
\hline Dosimetric Parameter & \multicolumn{5}{c}{ Average Value \pm StdDev/Gy } \\
\hline Mean dose to lung & $3.9 \pm 1.9$ & $4.0 \pm 1.5$ & $4.0 \pm 1.5$ & $4.0 \pm 1.5$ & $3.8 \pm 1.6$ \\
to ipsilateral lung & $6.2 \pm 2.7$ & $7.4 \pm 3.4$ & $7.5 \pm 3.3$ & $6.3 \pm 2.5$ & $5.7 \pm 2.6$ \\
to contralateral lung & $2.5 \pm 2.1$ & $1.4 \pm 1.1$ & $1.3 \pm 1.0$ & $2.0 \pm 1.1$ & $2.0 \pm 1.2$ \\
Mean dose to heart & $3.4 \pm 3.3$ & $3.0 \pm 2.0$ & $3.0 \pm 1.9$ & $2.7 \pm 3.0$ & $2.6 \pm 2.8$ \\
$\begin{array}{c}\text { Maximum dose to } \\
\text { spinal cord }\end{array}$ & $11.6 \pm 6.8$ & $12.8 \pm 7.2$ & $12.6 \pm 7.0$ & $14.4 \pm 5.5$ & $13.6 \pm 6.4$ \\
\hline
\end{tabular}

StdDev: standard deviation.

lower mean lung dose compared to the 3D techniques. There was no significant difference between the HT plans and the VMAT-1 plans, or between the HT plans and the VMAT-2 plans. However, the VMAT-2 plans showed significantly lower ipsilateral mean lung dose compared to the VMAT-1 plans $(\mathrm{p}=0.002)$.

Figure 2 plots the average RDV as a function of $D_{\mathrm{L} 50}$ using the S-shaped model, the logistic model, and the linear model, respectively. Paired t-tests were used to compare the RDV values among different models using the same treatment technique and $D_{\mathrm{L} 50}$. The logistic model gave significantly larger RDV values compared to the S-shaped model $(\mathrm{p}<0.05)$, and the linear model gave significantly larger RDV values compared to the other two models $(\mathrm{p}<0.05)$ over the evaluated range of $D_{\mathrm{L} 50}$. Although the HT and VMAT-2 plans showed lower average RDV values compared to the other treatment techniques with each of the three RDV models, the absolute difference is relatively small. Paried t-tests showed no significant difference in RDV values in all the five delivery techniques.

Table 4 lists average NTCP values for each delivery technique using the LKB model and the MLD model, respectively, at the prescription dose level of $50 \mathrm{~Gy}$. Similar to results obtained using the RDV models, the differences among the delivery techniques were not statistically significant using either the LKB model or the MLD model ( $p>0.05$ ). The NTCP values obtained using the LKB model were significantly larger than those obtained using the MLD model $(\mathrm{p}<0.05)$.

Figure 3 shows the RDV value as a function of mean lung dose (MLD) in treatment plans with each of the three RDV models. In general, the RDV increases with increasing MLD. The correlation between the MLD and the RDV was analyzed by evaluating Spearman's rank order correlation coefficients; the results showed strong correlation (correlation coefficient $>0.9$ ) with statistical significance ( $p$-value $<0.01$ ) for any given delivery technique and the RDV formulation used. Figure 4 shows the NTCP value as a function of mean lung dose with with the LKB and the MLD models, respectively. In general, both the RDV and the NTCP values increase with increasing MLD value. The correlation between the MLD and the NTCP value was analyzed by evaluating Spearman's rank order correlation coefficients; the results showed strong correlation (correlation coefficient $>0.95$ ) with statistical significance ( $p$-value $<0.01)$ for any 


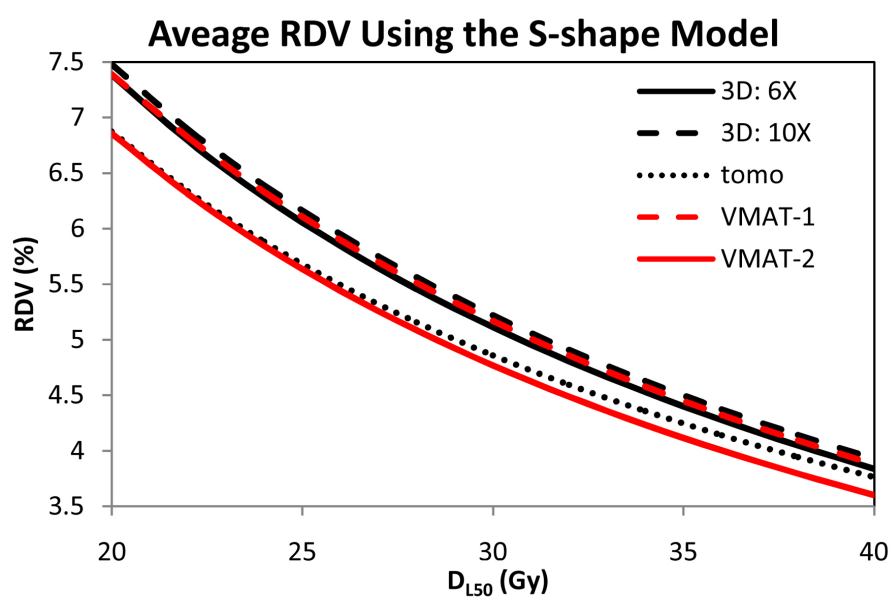

(a)

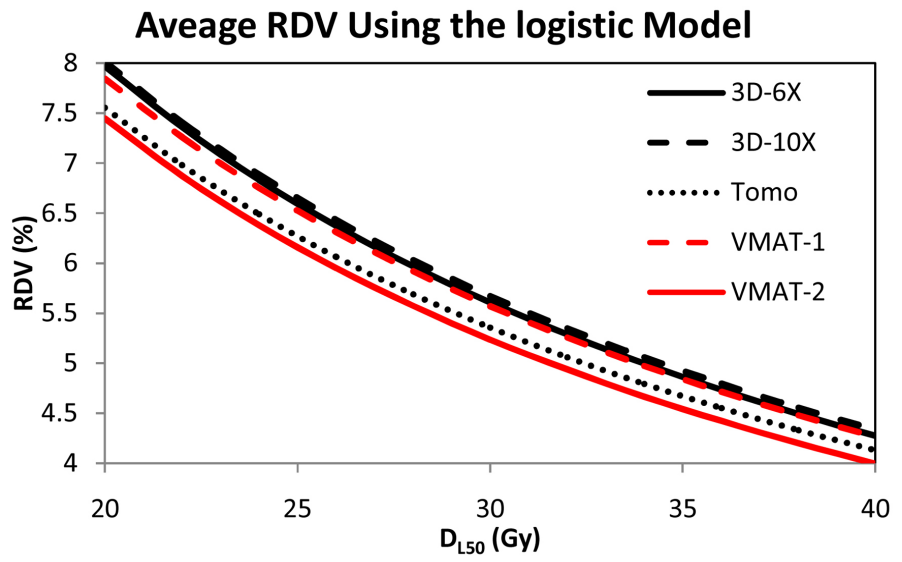

(b)

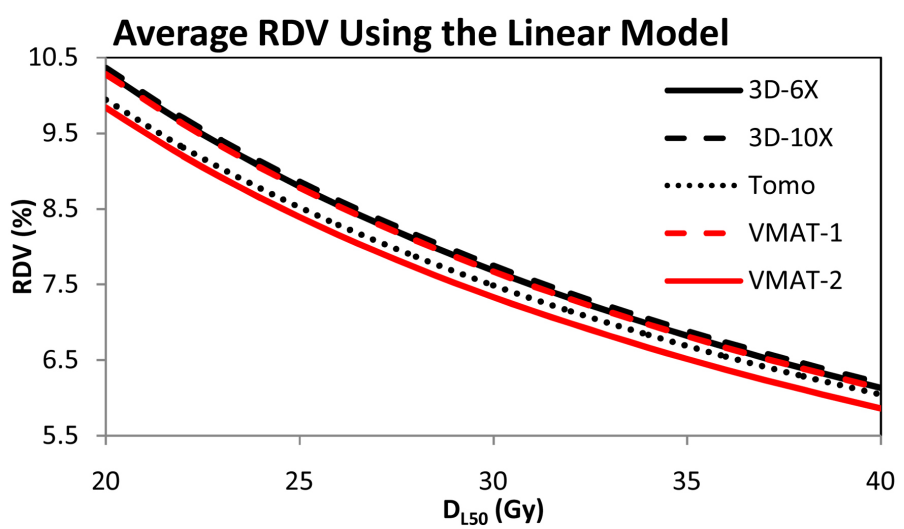

(c)

Figure 2. Comparison of average RDV values with each treatment delivery technique using the S-shaped model (a), the logistic model (b), and the linear model (c).

given delivery technique and the NTCP formulation used.

\section{Discussions}

Radiation pneumonitis (RP) is a primary concern in SBRT treatment of early stage non-small cell lung cancer. While most patients will develop asymptomatic 


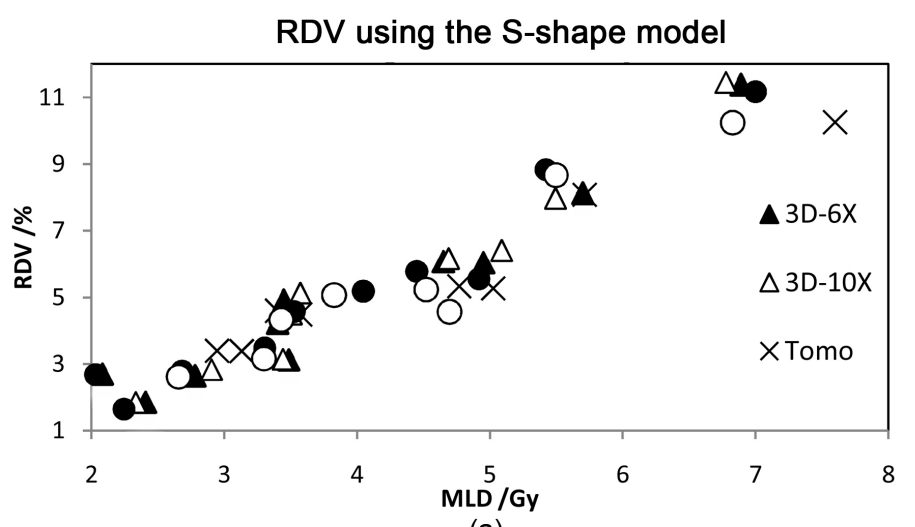

(a)

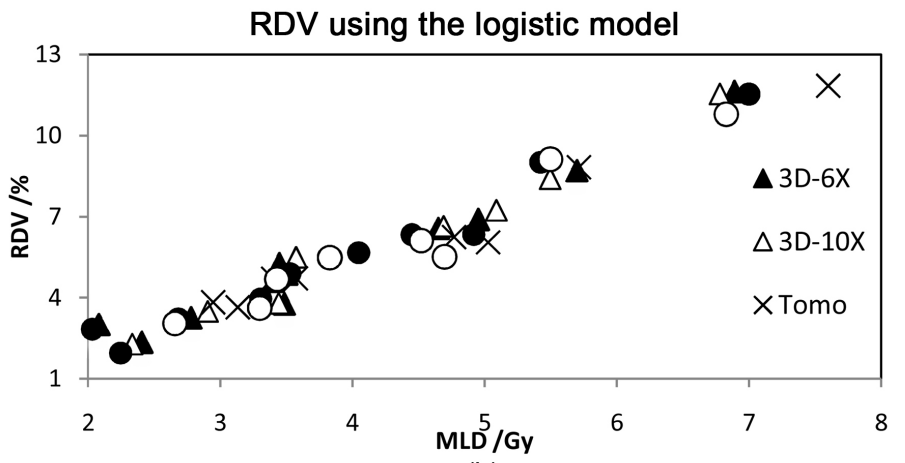

(b)

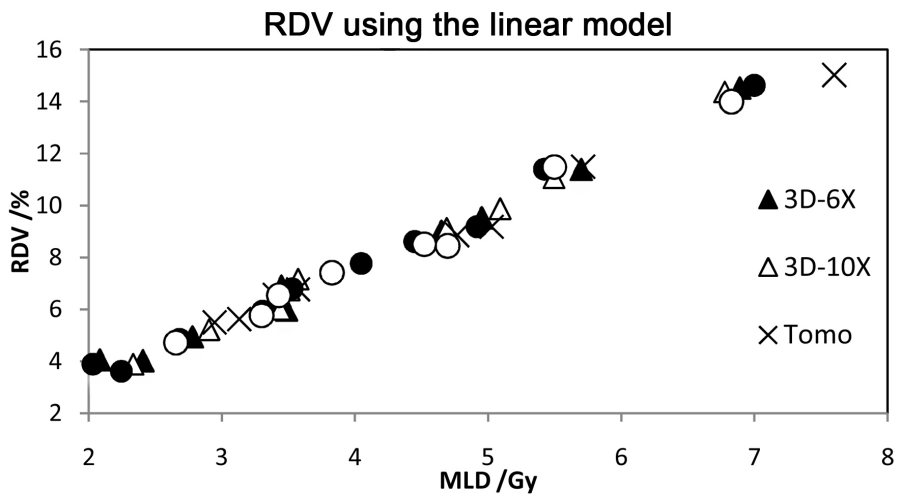

(c)

Figure 3. RDV values as a function of mean lung dose (MLD) with each treatment delivery technique using the S-shaped model (a), the logistic model (b), and the linear model (c), respectively.

Grade-1 RP, the chance of developing Grade-2 or 3 RP is relatively low [5] [15]. However, pulmonary toxicity is the primary type of late toxicity after SBRT treatments to the lung, and grade 5 pulmonary toxicities have been reported [5]. As patients can be potentially cured with SBRT treatments, it is imperative to quantitatively assess RP risks during SBRT treatment planning to avoid treatment-induced morbidities. Given hypofractionation scheme in SBRT treatments, normal lung response to radiation could be different from that in conventional RT treatments. However, most recent dosimetric planning studies still used conventional dosimetric parameters in evaluation of lung toxicity [16] [17]. 


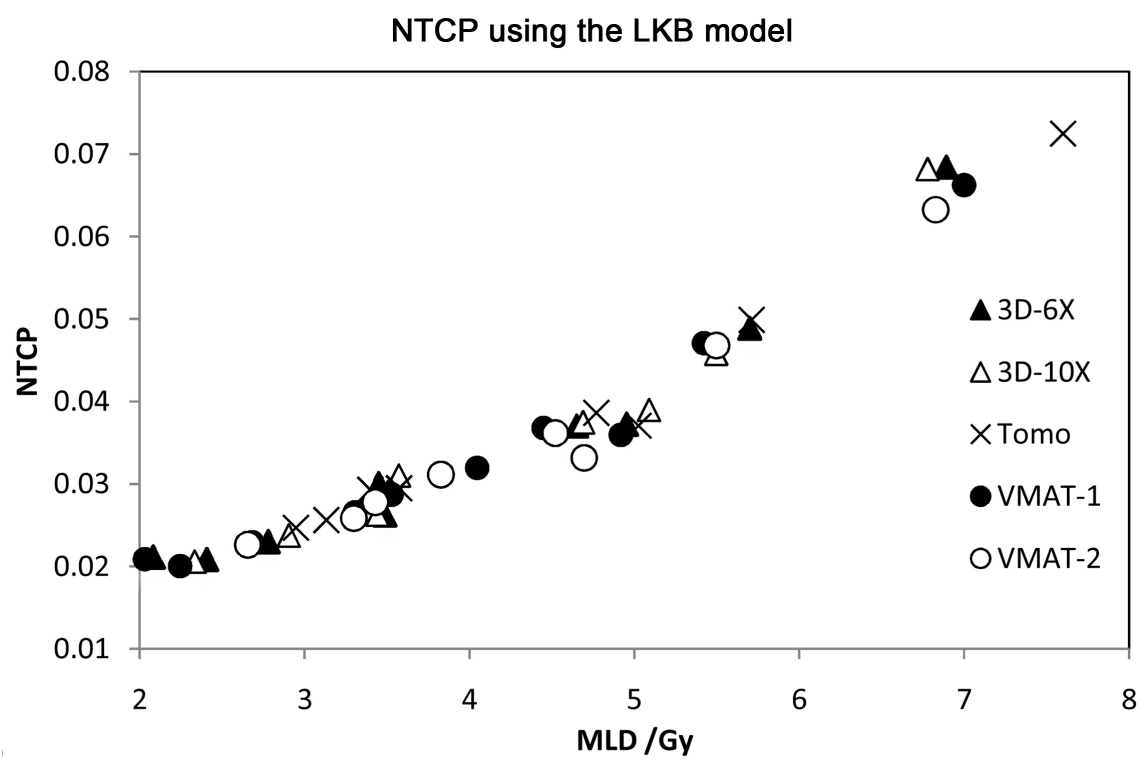

(a)

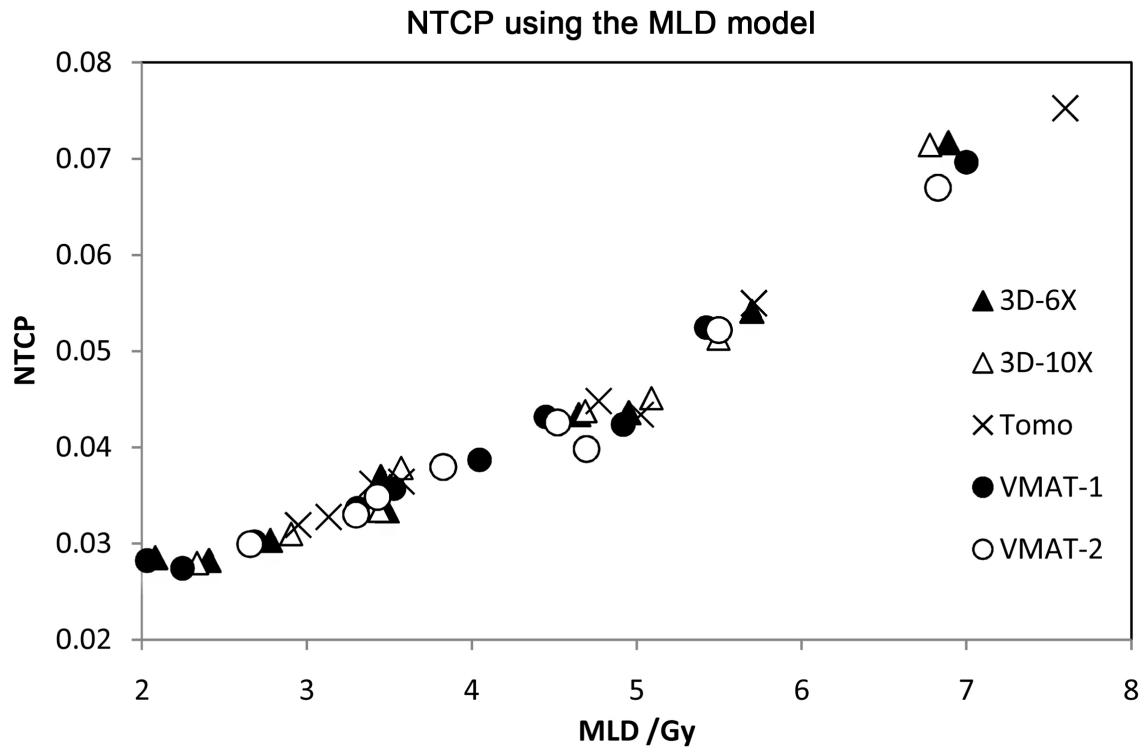

(b)

Figure 4. NTCP values as a function of mean lung dose (MLD) with each treatment delivery technique using the LKB model (a) and the MLD model (b), respectively.

We believe that this is the first study that used a comprehensive set of radiobiological models for comparison of multiple delivery techniques for SBRT treatment of early-stage NSCLC.

VMAT and HT are two modern IMRT techniques that utilize a rotating gantry to delivery radiation from a large number of beam portals. When the two techniques are used to deliver radiation to the thoracic region, a significantly higher percentage of the normal lung volume typically receives low dose radiation compared to 3D-CRT techniques, which has raised concerns over increased lung toxicities [18] [19]. Therefore, we were motivated to carry out this study to compare the VMAT and HT techniques with conventional 3D-CRT techniques 
Table 4. Average normal tissue complication probability with each delivery technique using the Lyman-Kutcher-Burman (LKB) model and the mean-lung-dose (MLD) model, respectively.

\begin{tabular}{ccc}
\hline \multirow{2}{*}{ Delivery Technique } & \multicolumn{2}{c}{ Average NTCP \pm StdDev $($ Range $)$} \\
\cline { 2 - 3 } & LKB Model & MLD Model \\
\hline 3D-6MV & $0.0341 \pm 0.0149$ & $0.0405 \pm 0.0136$ \\
& $(0.0209-0.0685)$ & $(0.0282-0.0717)$ \\
3D-10MV & $0.0342 \pm 0.0145$ & $0.0406 \pm 0.0132$ \\
& $(0.0206-0.0682)$ & $(0.0280-0.0714)$ \\
Tomo & $0.0346 \pm 0.0163$ & $0.0409 \pm 0.0149$ \\
& $(0.0182-0.0725)$ & $(0.0256-0.0753)$ \\
VMAT-1 & $0.0337 \pm 0.0141$ & $0.0402 \pm 0.0129$ \\
& $(0.0201-0.0662)$ & $(0.0274-0.0696)$ \\
VMAT-2 & $0.0326 \pm 0.0136$ & $0.0391 \pm 0.0125$ \\
& $(0.0190-0.0633)$ & $(0.0264-0.0670)$ \\
\hline
\end{tabular}

StdDev: standard deviation.

in terms of lung toxicities by using radiobiological models. Based on the results given by existing radiobiological models, the VMAT and HT techniques could at least achieve similar levels of lung sparing compared to 3D-CRT techniques.

The EUD and FSU models are two widely used normal tissue toxicity models. While both models reduce the normal organ DVH to a single parameter, they differ by representing the DVH with a dose parameter and a volume parameter, respectively.

For a comprehensive evaluation of lung toxicity with different delivery techniques, both models were used in this study. It is interesting to note that the results from both models showed remarkable agreement in this study, indicating similar predictive power with these two types of models.

Figure 3 and Figure 4 provided practical results relevant for clinical dosimetry planning in SBRT treatment of early-stage non-small lung cancer patients. Even with non-linear NTCP and RDV models, the degree of lung toxicities, as measured by the NTCP value or the RDV value, increases approximately monotonously with the mean lung dose. Note that since the normalized lung DVH was used in model calculations, the RDV value does not have a linear relationship with the mean lung dose even with the linear model. Based on results from the radiobiological models, the mean lung dose can be an efficient parameter to use in evaluation of lung toxicities in lung SBRT treatment plans.

Different dose normalization methods could affect results in studies that correlate dosimetric parameters with RP occurrence risks. Baker et al. evaluated a set of dosimetric and clinical parameters for correlations with RP after five-fraction SBRT treatments [20]. No DVH normalization was performed. While certain dosimetric parameters were found to be predictive of RP in univariate analysis, the correlations were not significant in multivariable analysis. Guckenberger et al. analyzed 59 patients who received SBRT treatments to the lung with various fractionation schemes [13]. The lung DVHs were normalized using $\alpha / \beta$ 
ratio of $3 \mathrm{~Gy}$. The MLD of the ipsilateral lung, as well as the ipsilateral lung volume exposed to doses between 2.5 and $50 \mathrm{~Gy}$, were found to be correlated to RP incidence. Scheenstra et al. evaluated the relation between local dose and relative lung perfusion reduction after SBRT treatment, using $\alpha / \beta$ ratio of 3 Gy to normalize the lung DVH [12]. The study found that the relation between local dose and perfusion reduction can be best modeled by a logistic function. The $k$ value in the logistic model was found to be 2.2 .

The value of $D_{\mathrm{L} 50}$ depends on the type of radiation response endpoints used, and in the case of RP, the grade of RP used in the analysis. Theuws et al. and Marks et al. studied radiation-induced lung perfusion reduction for patients who received radiation treatments in the thorax region including lymphoma and breast cancer patients [21] [22]. Their combined data gave $D_{\mathrm{L} 50}$ of $55 \mathrm{~Gy}$ and $k$ value of 2.2 in the logistic model. In contrast, Scheenstra et al. evaluated lung perfusion reduction for lung cancer patients receiving SBRT treatments, and they found $D_{\mathrm{L} 50}$ to be $28.7 \mathrm{~Gy}$ (95\% confidence interval (CI): $26.3-31.1$ ) and $k$ to be 2.2 (95\% CI: 1.8 - 2.5) in the logistic model (12). Marks et al. compiled clinical RP data (5). Using the MLD model and the LKB model to fit the data, $D_{\mathrm{L} 50}$ was found to be $30.8 \mathrm{~Gy}$ (95\% CI: 28.7 - 33.9) and 31.4 Gy (95\% CI: 29.0 - 34.7), respectively. It should be noted that heterogeneous RP criteria were used in the compiled data. Due to the uncertainty of $D_{\mathrm{L} 50}$ values in published results, we allowed $D_{L 50}$ to vary in the range of 20 to $40 \mathrm{~Gy}$ in this study.

\section{Conclusion}

Using different radiobiological models for radiation-induced lung toxicities, a comprehensive set of delivery techniques were compared for SBRT treatment of early-stage lung cancer. The current study showed that VMAT and HT plans could achieve comparable lung sparing compared to traditional 3D-CRT techniques. The NTCP modeling results confirmed the results based on parallel FSU models. However, the validity of the radiobiological models should be tested by clinical data.

\section{Compliance with Ethical Standards}

\section{Conflict of Interest}

The authors have no conflict of interest to declare.

\section{Resource of Funding}

The authors declare no funding resources used for this study.

\section{Research Involving Human Participants and/or Animals}

This study did not involve human participants and/or animals.

\section{Informed Consent}

Informed consent was not applicable in this study. 


\section{References}

[1] Chang, B.K. and Timmerman, R.D. (2007) Stereotactic Body Radiation Therapy: A Comprehensive Review. American Journal of Clinical Oncology, 30, 637-644. https://doi.org/10.1097/COC.0b013e3180ca7cb1

[2] Timmerman, R., Paulus, R., Galvin, J., et al. (2010) Stereotactic Body Radiation Therapy for Inoperable Early Stage Lung Cancer. JAMA, 303, 1070-1076. https://doi.org/10.1001/jama.2010.261

[3] Baker, S., Dahele, M., Lagerwaard, F.J. and Senan, S. (2016) A Critical Review of Recent Developments in Radiotherapy for Non-Small Cell Lung Cancer. Radiation Oncology, 11, 115. https://doi.org/10.1186/s13014-016-0693-8

[4] Chi, A., Liao, Z., Nguyen, N.P., et al. (2010) Systematic Review of the Patterns of Failure Following Stereotactic Body Radiation Therapy in Early-Stage Non-SmallCell Lung Cancer: Clinical Implications. Radiotherapy and Oncology, 94, 1-11. https://doi.org/10.1016/j.radonc.2009.12.008

[5] Marks, L.B., Bentzen, S.M., Deasy, J.O., et al. (2010) Radiation Dose-Volume Effects in the Lung. International Journal of Radiation Oncology * Biology * Physics, 76, S70-S76. https://doi.org/10.1016/j.ijrobp.2009.06.091

[6] Jin, J., Kong, F., Chetty, I.J., et al. (2010) Impact of Fraction Size on Lung Radiation Toxicity: Hypofractionation May Be Beneficial in Dose Escalation of Radiotherapy for Lung Cancers. International Journal of Radiation Oncology ${ }^{\star}$ Biology ${ }^{*}$ Physics, 76, 782-788. https://doi.org/10.1016/j.ijrobp.2009.02.079

[7] Niemierko, A. (1997) Reporting and Analyzing Dose Distributions: A Concept of Equivalent Uniform Dose. Medical Physics, 24, 103-110. https://doi.org/10.1118/1.598063

[8] Jackson, A., Kutcher, G.J., Yorke, E.D., et al. (1993) Probability of Radiation-Induced Complications for Normal Tissue with Parallel Architecture Subject to NonUniform Irradiation. Medical Physics, 20, 613-625. https://doi.org/10.1118/1.597056

[9] Yorke, E.D., Kutcher, G.J., Jackson, A. and Ling, C.C. (1993) Probability of Radiation-Induced Complications in Normal Tissues with Parallel Architecture under Conditions of Uniform Whole or Partial Organ Irradiation. Radiotherapy and Oncology, 26, 226-237. https://doi.org/10.1016/0167-8140(93)90264-9

[10] Jackson, A., Ten Haken, R.K., Robertson, J.M., et al. (1995) Analysis of Clinical Complication Data for Radiation Hepatitis Using a Parallel Architecture Model. International Journal of Radiation Oncology ${ }^{*}$ Biology ${ }^{*}$ Physics, 31, 883-891. https://doi.org/10.1016/0360-3016(94)00471-4

[11] Kwa, S.L.S., Theuws, J.C.T., Wagenaar, A., et al. (1998) Evaluation of Two DoseVolume Histogram Reduction Models for the Prediction of Radiation Pneumonitis. Radiotherapy and Oncology, 48, 61-69. https://doi.org/10.1016/S0167-8140(98)00020-6

[12] Scheenstra, A.E.H., Rossi, M.M.G., Belderbos, J.S.A., et al. (2013) Local Dose-Effect Relations for Lung Perfusion Post Stereotactic Body Radiotherapy. Radiotherapy and Oncology, 107, 398-402. https://doi.org/10.1016/j.radonc.2013.04.003

[13] Guckenberger, M., Baier, K., Polat, B., et al. (2010) Dose-Response Relationship for Radiation-Induced Pneumonitis after Pulmonary Stereotactic Body Radiotherapy. Radiotherapy and Oncology, 97, 65-70. https://doi.org/10.1016/j.radonc.2010.04.027

[14] R Core Team (2013) R: A Language and Environment for Statistical Computing. R Foundation for Statistical Computing, Vienna. http://www.R-project.org/ 
[15] Yamashita, H., Takahashi, W. and Haga, A. and Nakagawa, K. (2014) Radiation Pneumonitis after Stereotactic Radiation Therapy for Lung Cancer. World Journal of Radiology, 6, 708-715. https://doi.org/10.4329/wjr.v6.i9.708

[16] Ong, C.L., Verbakel, W.F.A.R., Cuijpers. J.P., et al. (2010) Stereotactic Radiotherapy for Peripheral Lung Tumors: A Comparison of Volumetric Modulated Arc Therapy with 3 Other Delivery Techniques. Radiotherapy and Oncology, 97, 437-442. https://doi.org/10.1016/j.radonc.2010.09.027

[17] McGrath, S.D., Matuszak, M.M., Yan, D., et al. (2010) Volumetric Modulated Arc Therapy for Delivery of Hypofractionated Stereotactic Lung Radiotherapy: A Dosimetric and Treatment Efficiency Analysis. Radiotherapy and Oncology, 95, 153 157. https://doi.org/10.1016/j.radonc.2009.12.039

[18] Jo, I., Kay, C., Kim, J., et al. (2010) Significance of Low-Dose Radiation Distribution in Development of Radiation Pneumonitis after Helical-Tomotherapy-Based Hypofractionated Radiotherapy for Pulmonary Metastases. Journal of Radiation Research, 55, 105-112. https://doi.org/10.1093/jrr/rrt080

[19] Khalil, A.A., Hoffmann, L., Moeller, D.S., et al. (2015) New Dose Constraint Reduces Radiation-Induced Fatal Pneumonitis in Locally Advanced Non-Small Cell Lung Cancer Patients Treated with Intensity-Modulated Radiotherapy. Acta Oncologica, 54, 1343-1349. https://doi.org/10.3109/0284186X.2015.1061216

[20] Baker, R., Han, G., Sarangkasiri, S., et al. (2013) Clinical and Dosimetric Predictors of Radiation Pneumonitis in a Large Series of Patients Treated with Stereotactic Radiation Therapy to the Lung. International Journal of Radiation Oncology Biology Physics, 85, 190-195. https://doi.org/10.1016/j.ijrobp.2012.03.041

[21] Theuws, J.C.M., Kwa, S.L.S., Wagenaar, A.C., et al. (1998) Dose-Effect Relations for Early Local Pulmonary Injury after Irradiation for Malignant Lymphoma and Breast Cancer. Radiotherapy and Oncology, 48, 33-43. https://doi.org/10.1016/S0167-8140(98)00019-X

[22] Marks, L.B., Munley, M.T., Spencer, D.P., et al. (1997) Quantification of Radiation-Induced Regional Lung Injury with Perfusion Imaging. International Journal of Radiation Oncology Biology Physics, 38, 399-409. https://doi.org/10.1016/S0360-3016(97)00013-8 